Bull. Korean Math. Soc. 51 (2014), No. 3, pp. 653-657

http://dx.doi.org/10.4134/BKMS.2014.51.3.653

\title{
RESULTS OF CERTAIN LOCAL COHOMOLOGY MODULES
}

\author{
Amir Mafi and Atiyeh Pour Eshmanan Talemi
}

Abstract. Let $R$ be a commutative Noetherian ring, $I$ and $J$ two ideals of $R$, and $M$ a finitely generated $R$-module. We prove that

$$
\operatorname{Ext}_{R}^{i}\left(R / I, H_{I, J}^{t}(M)\right)
$$

is finitely generated for $i=0,1$ where $t=\inf \left\{i \in \mathbb{N}_{0}: H_{I, J}^{i}(M)\right.$ is not finitely generated $\}$. Also, we prove that $H_{I+J}^{i}\left(H_{I, J}^{t}(M)\right)$ is Artinian when $\operatorname{dim}(R / I+J)=0$ and $i=0,1$.

\section{Introduction}

Throughout this paper, we assume that $R$ is a commutative Noetherian ring with non-zero identity, $I$ and $J$ two ideals of $R$, and $M$ a finitely generated $R$-module. Recently Takahashi, Yoshino and Yoshizawa in [18], introduce the module $H_{I, J}^{i}(M)$ as a generalization of the ordinary local cohomology module $H_{I}^{i}(M)$ that defined by Grothendieck in [5]. They considered $(I, J)$ torsion submodule $\Gamma_{I, J}(M)$ of $M$ which consists of all elements $x$ of $M$ with $\operatorname{Supp}(R x) \subseteq W(I, J)$, where $W(I, J)=\left\{\mathfrak{p} \in \operatorname{Spec}(R): I^{n} \subseteq \mathfrak{p}+J\right.$ for an integer $n \geq 1\}$. Furthermore, they defined the local cohomology functor $H_{I, J}^{i}$ with respect to $(I, J)$ to be the $i$-th right derived functor of $\Gamma_{I, J}$. Notice that if $J=0$, then $H_{I, J}^{i}$ coincides with the ordinary local cohomology functor $H_{I}^{i}$. In [6], Grothendieck conjectured that the module $\operatorname{Hom}_{R}\left(R / I, H_{I}^{i}(M)\right)$ is finitely generated for all $i \geq 0$. Hartshorne provided a counter-example to this conjecture in [7]. However, this conjecture is true in many cases see for example [1], [3], [4], [11] and [12]. In [8], Huneke asked when the local cohomology module $H_{I}^{i}(M)$ is Artinian. In this regard see [9], [10], [13] and [17]. The main aim of this paper is to prove the following theorem.

Theorem 1.1. Let $t$ be a non-negative integer such that $H_{I, J}^{i}(M)$ is finitely generated for all $i<t$. Then the following statements hold:

(a) $\operatorname{Ext}^{j}\left(R / I, H_{I, J}^{t}(M)\right)$ is finitely generated for $j=0,1$.

(b) $H_{I+J}^{i}\left(H_{I, J}^{t}(M)\right)$ is Artinian when $\operatorname{dim}(R / I+J)=0$ and $i=0,1$.

Received May 30, 2012.

2010 Mathematics Subject Classification. 13D45, 13E99.

Key words and phrases. local cohomology, Artinian modules. 


\section{The results}

Recall that $\tilde{W}(I, J)$ is called the set of ideals $\mathfrak{a}$ of $R$ such that $I^{n} \subseteq \mathfrak{a}+J$ for some integer $n$ (cf. [18]).

Lemma 2.1. Let $\mathfrak{a}$ be an ideal in $\tilde{W}(I, J)$. Then $\left(0:_{M} \mathfrak{a}\right)=\left(0:_{\Gamma_{I, J}(M)} \mathfrak{a}\right)$.

Proof. Since $\Gamma_{I, J}(M) \subseteq M$, we have $\left(0:_{\Gamma_{I, J}(M)} \mathfrak{a}\right) \subseteq\left(0::_{M} \mathfrak{a}\right)$. To prove the converse inclusion, take $x \in(0: M \mathfrak{a})$, then $\mathfrak{a} \subseteq \operatorname{Ann}(x)$ and there is an integer $n$ with $I^{n} \subseteq \mathfrak{a}+J$. Thus $I^{n} \subseteq \operatorname{Ann}(x)+J$ and hence $x \in\left(0:_{\Gamma_{I, J}(M)} \mathfrak{a}\right)$, as required.

The following theorem extends [4, Theorem A].

Theorem 2.2. Let $\mathfrak{a}$ be an ideal in $\tilde{W}(I, J), t$ a non-negative integer, and $N$ an $R$-module such that $\operatorname{Ext}_{R}^{i}(R / \mathfrak{a}, N)$ is a finitely generated $R$-module for $i=$ $t, t+1$. If $H_{I, J}^{i}(N)$ is finitely generated for all $i<t$, then $\operatorname{Ext}_{R}^{j}\left(R / \mathfrak{a}, H_{I, J}^{t}(N)\right)$ is finitely generated for $j=0,1$.

Proof. We use induction on $t$. Let $t=0$. Then the short exact sequence

$$
0 \longrightarrow \Gamma_{I, J}(N) \longrightarrow N \longrightarrow N / \Gamma_{I, J}(N) \longrightarrow 0
$$

induces the following exact sequence

$$
0 \longrightarrow \operatorname{Hom}_{R}\left(R / \mathfrak{a}, \Gamma_{I, J}(N)\right) \longrightarrow \operatorname{Hom}_{R}(R / \mathfrak{a}, N) \longrightarrow \operatorname{Hom}_{R}\left(R / \mathfrak{a}, N / \Gamma_{I, J}(N)\right)
$$

$$
\longrightarrow \operatorname{Ext}_{R}^{1}\left(R / \mathfrak{a}, \Gamma_{I, J}(N)\right) \longrightarrow \operatorname{Ext}_{R}^{1}(R / \mathfrak{a}, N) .
$$

By Lemma 2.1 and [18, Corollary 1.13], we have $\operatorname{Hom}_{R}\left(R / \mathfrak{a}, N / \Gamma_{I, J}(N)\right)=0$. Hence by our hypothesis and using $(\dagger \dagger)$ the result follows. Suppose that $t>0$ and that the case $t-1$ is settled. Since $\Gamma_{I, J}(N)$ is finitely generated, the $R$ module $\operatorname{Ext}_{R}^{i}\left(R / \mathfrak{a}, \Gamma_{I, J}(N)\right)$ is finitely generated for all $i$. Now, by the exact sequence $(\dagger), \operatorname{Ext}_{R}^{i}\left(R / \mathfrak{a}, N / \Gamma_{I, J}(N)\right)$ is finitely generated for $i=t, t+1$. On the other hand $\Gamma_{I, J}\left(N / \Gamma_{I, J}(N)\right)=0$ and $H_{I, J}^{i}(N) \cong H_{I, J}^{i}\left(N / \Gamma_{I, J}(N)\right)$ for all $i>0$. Hence we may assume that $\Gamma_{I, J}(N)=0$. Let $E$ be an injective hull of $N$ and put $L=E / N$. Thus $\Gamma_{I, J}(E)=0$ and $\operatorname{Hom}_{R}(R / \mathfrak{a}, E)=0$. Consequently, $\operatorname{Ext}_{R}^{i}(R / \mathfrak{a}, L) \cong \operatorname{Ext}_{R}^{i+1}(R / \mathfrak{a}, N)$ and $H_{I, J}^{i}(L) \cong H_{I, J}^{i+1}(N)$ for all $i \geq 0$. Now the induction hypothesis yields that $\operatorname{Ext}_{R}^{j}\left(R / \mathfrak{a}, H_{I, J}^{t-1}(L)\right)$ is finitely generated for $j=0,1$, and hence $\operatorname{Ext}_{R}^{j}\left(R / \mathfrak{a}, H_{I, J}^{t}(N)\right)$ is finitely generated for $j=0,1$.

The following corollary immediately follows by Theorem 2.2.

Corollary 2.3. Let $t$ be a non-negative integer such that $H_{I, J}^{i}(M)$ is finitely generated for all $i<t$. Then $\operatorname{Ext}_{R}^{j}\left(R / I, H_{I, J}^{t}(M)\right)$ is finitely generated for $j=0,1$. 
Recall that an $R$-module $N$ is called Minimax if there is a finitely generated submodule $L$ of $N$ such that $N / L$ is Artinian (cf. [19]). The class of Minimax modules includes all finitely generated and all Artinian modules. Moreover, it is closed under taking submodules, quotients and extensions, i.e., it is a Serre subcategory of the category of $R$-modules (cf. [16] and [19]).

Proposition 2.4. Let $\mathfrak{a}$ be an ideal in $\tilde{W}(I, J), t$ a non-negative integer, and $N$ an $R$-module such that $\operatorname{Ext}_{R}^{i}(R / \mathfrak{a}, N)$ is a Minimax $R$-module for $i=t, t+1$. If $H_{I, J}^{i}(N)$ is Minimax for all $i<t$, then $\operatorname{Ext}_{R}^{j}\left(R / \mathfrak{a}, H_{I, J}^{t}(N)\right)$ is Minimax for $j=0,1$.

Proof. By using Minimax instead of finitely generated in the proof of Theorem 2.2 and using the same arguments the result follows.

Proposition 2.5. Let $t$ be a non-negative integer such that $H_{I, J}^{i}(M)$ is $A r$ tinian for all $i<t$. Then $H_{I}^{i}(M)$ is Artinian for all $i<t$.

Proof. Let $E^{\bullet}$ denote an injective resolution of the $R$-module $M$. By [18, Proposition 1.4], there is the following isomorphism of complexes

$$
\Gamma_{I}\left(\Gamma_{I, J}\left(E^{\bullet}\right)\right) \cong \Gamma_{I}\left(E^{\bullet}\right) .
$$

Hence, by [15, Theorem 11.38], there is the Grothendieck spectral sequence

$$
E_{2}^{p, q}:=H_{I}^{p}\left(H_{I, J}^{q}(M)\right) \underset{p}{\Longrightarrow} H_{I}^{p+q}(M) .
$$

Since $E_{i}^{p, q}$ is a subquotient of $E_{2}^{p, q}$ for all $i \geq 2$, by [2, Theorem 6.1.2] we deduce that $E_{i}^{p, q}=0$ for all $i \geq 2, p \geq 1$, and $q<t$. There is a finite filtration of the module $H^{j}=H_{I}^{j}(M)$

$$
0=\varphi^{j+1} H^{j} \subseteq \varphi^{j} H^{j} \subseteq \cdots \subseteq \varphi^{1} H^{j} \subseteq \varphi^{0} H^{j}=H_{I}^{j}(M)
$$

such that $E_{\infty}^{i, j-i} \cong \varphi^{i} H^{j} / \varphi^{i+1} H^{j}$ for all $0 \leq i \leq j$. Since $E_{i}^{p, q} \cong E_{\infty}^{p, q}$ for $i$ sufficiently large, we have that $E_{\infty}^{p, q}=0$ for all $p \geq 1$ and $q<t$. Hence, from (*) we conclude that $0=\varphi^{j+1} H^{j}=\varphi^{j} H^{j}=\cdots=\varphi^{1} H^{j}$ and $E_{\infty}^{0, j} \cong H_{I}^{j}(M)$ for all $j<t$. On the other hand $E_{\infty}^{0, j} \cong E_{2}^{0, j}$ for all $j<t$. Hence, we get that $H_{I}^{i}(M)$ is Artinian for all $i<t$.

Corollary 2.6. The following holds:

$$
\inf \left\{i \mid H_{I, J}^{i}(M) \text { is not Artinian }\right\} \leq \inf \left\{i \mid H_{I}^{i}(M) \text { is not Artinian }\right\} .
$$

Proof. This is immediate by Proposition 2.5.

Theorem 2.7. Let $t$ be a non-negative integer such that $H_{I, J}^{i}(M)$ is finitely generated for all $i<t$. If $\operatorname{dim} R / I+J=0$, then $H_{I+J}^{j}\left(H_{I, J}^{t}(M)\right)$ is Artinian for $j=0,1$. 
Proof. By [18, Proposition 1.4] and [15, Theorem 11.38], there is the Grothendieck spectral sequence

$$
E_{2}^{p, q}:=H_{I^{\prime}}^{p}\left(H_{I, J}^{q}(M)\right) \underset{p}{\Longrightarrow} H_{I+J}^{p+q}(M) .
$$

For all $i \geq 2$ and $j=0,1$, we consider the exact sequence

$$
0 \longrightarrow \operatorname{ker} d_{i}^{j, t} \longrightarrow E_{i}^{j, t} \stackrel{d_{i}^{j, t}}{\longrightarrow} E_{i}^{i+j, t-i+1} .
$$

Since $E_{i}^{j, t}=\operatorname{ker} d_{i-1}^{j, t} / \operatorname{im} d_{i-1}^{j-i+1, t+i-2}$ and $E_{i}^{p, q}=0$ for all $q<0$, we may use (*) to obtain $E_{t+2}^{j, t} \cong E_{t+3}^{j, t} \cong \cdots \cong E_{\infty}^{j, t}$ and $E_{i+1}^{j, t} \cong \operatorname{ker} d_{i}^{j, t}$. There exists a finite filtration of the module $H^{t+j}=H_{I+J}^{t+j}(M)$,

$$
0=\varphi^{t+j+1} H^{t+j} \subseteq \varphi^{t+j} H^{t+j} \subseteq \cdots \subseteq \varphi^{1} H^{t+j} \subseteq \varphi^{0} H^{t+j}=H_{I+I^{\prime}}^{t+j}(M)
$$

such that $E_{\infty}^{i, t+j-1}=\varphi^{i} H^{t+j} / \varphi^{i+1} H^{t+j}$ for all $0 \leq i \leq t+j$. Therefore ker $d_{t+1}^{j, t}$ is Artinian and notice that $E_{i}^{p, q}$ is a subquotient of $E_{2}^{p, q}$ for all $i \geq 2$. Hence by the exact sequence $(\star)$ the result follows, as required.

The following corollary improves [17, Theorem 2.2].

Corollary 2.8. If $\operatorname{dim}(R / I+J)=0$, then $H_{I+J}^{j}\left(H_{I, J}^{1}(M)\right)$ is Artinian for $j=0,1$.

Proof. This is immediate by Theorem 2.7.

Hartshorne [7] defined that an $R$-module $N$ is $I$-cofinte, if $\operatorname{Supp}(N) \subseteq V(I)$ and $\operatorname{Ext}_{R}^{i}(R / I, N)$ is a finitely generated $R$-module for all $i$.

The following result extends [14, Proposition 3.15].

Proposition 2.9. If $I^{\prime}$ is an ideal of $R$ such that $\operatorname{cd} I^{\prime}=1$ and $I \subseteq I^{\prime}$, then $H_{I^{\prime}}^{i}\left(H_{I, J}^{j}(M)\right)$ is $I^{\prime}$-cofinite for all $i$ and $j$, where cd is the cohomological dimension of $I^{\prime}$ in $R$.

Proof. By [18, Proposition 1.4] and [15, Theorem 11.38], there is the Grothendieck spectral sequence

$$
E_{2}^{p, q}:=H_{I^{\prime}}^{p}\left(H_{I, J}^{q}(M)\right) \underset{p}{\Longrightarrow} H_{I^{\prime}}^{p+q}(M) .
$$

By using the same arguments as in the proof of Theorem 2.7, for all $i \geq 0$ and all $j \geq 0$, we obtain the exact sequence

$$
0 \longrightarrow \operatorname{ker} d_{2}^{j, i} \longrightarrow E_{2}^{j, i} \stackrel{d_{2}^{j, i}}{\longrightarrow} E_{2}^{j+2, i-1} \longrightarrow \operatorname{coker} d_{2}^{j+2, i-1} \longrightarrow 0 .
$$

Since $H_{I^{\prime}}^{j}\left(H_{I, J}^{i}(M)\right)=E_{2}^{j, i}=0$ for all $j \geq 2$ and all $i \geq 0$, we can assume that $j=0,1$. Hence we have $E_{\infty}^{j, i} \cong \operatorname{ker} d_{2}^{j, i}=E_{2}^{j, i}$ for all $i \geq 0$. On the other hand $E_{\infty}^{j, i}=0$ for all $j \geq 2$ and so there is an exact sequence

$$
0 \longrightarrow H_{I^{\prime}}^{1}\left(H_{I, J}^{i-1}(M)\right) \longrightarrow H_{I^{\prime}}^{i}(M) \longrightarrow H_{I^{\prime}}^{0}\left(H_{I, J}^{i}(M)\right) \longrightarrow 0 .
$$

By our assumption $H_{I^{\prime}}^{i}(M)=0$ for all $i \neq 0,1$, and therefore by [14, Corollary $3.16]$ the result follows, as required. 


\title{
References
}

[1] J. Asadollahi, K. Khashyarmanesh, and Sh. Salarian, A generalization of the cofiniteness problem in local cohomology modules, J. Aust. Math. Soc. 75 (2003), no. 3, 313-324.

[2] M. P. Brodmann and R. Y. Sharp, Local Cohomology: An Algebraic Introduction with Geometric Applications, Cambridge Studies in Advanced Mathematics, 60. Cambridge University Press.

[3] D. Delfino and T. Marley, Cofinite modules and local cohomology, J. Pure Appl. Algebra 121 (1997), no. 1, 45-52.

[4] M. T. Dibaei and S. Yassemi, Finiteness of extension functors of local cohomology modules, Comm. Algebra 34 (2006), no. 8, 3097-3101.

[5] A. Grothendieck, Local Cohomology, Notes by R. Harthsorne, Lecture Notes in Math., 862, Springer-Verlag, 1966.

[6] _ Cohomologie locale des faisceaux cohérents et théorèmes de Lefschetz locaux et globaux, North-Holland, Amsterdam, 1968.

[7] R. Hartshorne, Affine duality and cofiniteness, Invent. Math. 9 (1970), 145-164.

[8] C. Huneke, Problems on local cohomology, Free resolutions in commutative algebra and algebraic geometry (Sundance, UT, 1990), 93-108, Res. Notes Math., 2, Jones and Bartlett, Boston, MA, 1992.

[9] R. Lü and Z. Tang, The f-depth of an ideal on a module, Proc. Amer. Math. Soc. 130 (2001), 1905-1912.

[10] A. Mafi, Some results on local cohomology modules, Arch. Math. (Basel) 87 (2006), no. $3,211-216$

[11] On the finiteness results of the generalized local cohomology modules, Algebra Colloq. 16 (2009), no. 2, 325-332.

[12] A generalization of the finiteness problem in local cohomology modules, Proc. Indian Acad. Sci. Math. Sci. 119 (2009), no. 2, 159-164.

[13] L. Melkersson, Some applications of a criterion for Artinianness of a module, J. Pure Appl. Algebra 101 (1995), no. 3, 291-303.

[14] _ Modules cofinite with respect to an ideal, J. Algebra 285 (2005), no. 2, 649-668.

[15] J. Rotman, Introduction to Homological Algebra, Academic Press, 1979.

[16] P. Rudlof, On minimax and related modules, Canad. J. Math. 44 (1992), no. 1, 154-166.

[17] R. Sazeedeh, Finiteness of graded local cohomology modules, J. Pure Appl. Algebra 212 (2008), no. 1, 275-280.

[18] R. Takahashi, Y. Yoshino, and T. Yoshizawa, Local cohomology based on a nonclosed support defined by a pair of ideals, J. Pure Appl. Algebra 213 (2009), no. 4, 582-600.

[19] H. Zöschinger, Minimax moduln, J. Algebra 102 (1986), no. 1, 1-32.

\author{
AMIR MAFi \\ University of Kurdistan Pasdaran ST. \\ P.O. Box: 416, SANANDAJ, IrAN \\ E-mail address: a_mafi@ipm.ir \\ Atiyeh Pour Eshmanan Talemi \\ Islamic Azad University SCIENCE AND Research BranCH \\ P.O. Box 14515-775, TEHRAN, IrAN \\ E-mail address: ptalemy@yahoo.com
}

